\title{
Benchmarking atomic data for astrophysics: Fe XVII X-ray lines
}

\begin{abstract}
G. Del Zanna
DAMTP, Centre for Mathematical Sciences, Wilberforce road Cambridge CB3 0WA, UK

e-mail: g.del-zanna@damtp.cam.ac.uk

Received 18 May 2011 / Accepted 30 September 2011

\section{ABSTRACT}

Recent R-matrix scattering calculations for electron impact excitation of Fe XvII are benchmarked against various X-ray solar observations. Quiescent active region observations are considered, together with a few SMM/FCS observations of active regions and flares analysed here. Extremely good agreement (within 10\%) between expected and measured line intensities is found, with the exception of a few weaker lines that appear to be blended, and without the need to invoke opacity effects in the stronger line. The long-standing discrepancies that have been present when distorted wave (DW) calculations are adopted are now finally resolved. As was shown in the Fe xvIII case, the differences with the DW calculations are caused by resonances that significantly increase the collision strengths of the $2 \mathrm{p}-3 \mathrm{~s}$ transitions. The strong Fe xvII lines can now be reliably used to measure electron temperatures in the solar corona and other astrophysical sources. Emission measure modelling with the most recent atomic data of quiescent active region cores indicates $\mathrm{O} / \mathrm{Fe}$ and $\mathrm{Ne} / \mathrm{Fe}$ abundances reduced by factors of two to four compared to the latest compilation of "photospheric" abundances.
\end{abstract}

Key words. techniques: spectroscopic - Sun: flares - Sun: corona - atomic data - line: identification

\section{Introduction}

This paper is one of a series in which atomic data and line identifications are benchmarked against experimental data (Del Zanna et al. 2004). Because of its peculiar atomic structure, Fe xvII produces just a few but very strong lines in the X-rays. They are normally the dominant lines in any X-ray spectrum of the Sun, and are also prominent in a wide range of astrophysical sources. Solar and laboratory observations have always shown, until recently, large discrepancies between predicted and observed line intensities, with deviations of factors of two or more. A large amount of literature exists on the subject, where authors have tried different ways to explain the discrepancies. Only a few solar papers are mentioned in this paper, while the extensive literature on laboratory measurements (often reaching contradictory conclusions) is not discussed.

Until recently, only distorted-wave (DW) calculations have been available. The most used over a long period of time have been those of Bhatia \& Doschek (1992), which included a target with 37 levels. The suites of codes developed at UCL were used for the calculations. Bhatia \& Saba (2001) improved on Bhatia \& Doschek (1992) by performing an extensive set of DW calculations adopting different targets, the largest one including 73 levels. Small improvements (of the order of 10\%) in the predicted vs. observed line intensities were obtained with the largest target.

As we see later, the DW atomic data suggest that the strongest Fe XVII line (at 15.0 ̊) has an observed intensity much weaker than predicted, so many studies have been performed to explain the discrepancies as an opacity effect (since the strongest line has a high oscillator strength). See, for example, Bhatia \& Saba (2001). Different authors have reached opposite conclusions, however. For example, Saba et al. (1999) used the Solar Maximum Mission (SMM) flat crystal spectrometer (FCS) data and found a significant centre-to-limb variation, that was interpreted as an indication of a strong opacity effect in the strongest line. However, Phillips et al. (1996), from the same observations, found an opposite centre-to-limb trend.

It is interesting to note that exactly the same large discrepancies were present for Fe XVIII (in particular for the $2 s^{2} 2 p^{4} 3 s \rightarrow$ $2 s^{2} 2 p^{5}$ transitions), although they did not receive much attention in the literature, perhaps because these lines are weaker than the Fe XVII ones. The problem was only solved when the first R-matrix calculations for this ion were performed by Witthoeft et al. (2006). As shown in Witthoeft et al. (2006) with detailed analysis and comparisons, the main reason for the improved results was that the collision strengths to the $2 \mathrm{~s}^{2} 2 \mathrm{p}^{4} 3 \mathrm{~s}$ levels were strongly enhanced by the resonances. Excellent agreement between predicted and observed intensities was finally found, as discussed in detail in Del Zanna (2006), where the potential use of the Fe xvIII lines to measure electron densities in laboratory plasmas and temperatures for a wide range of astrophysical sources was presented for the first time.

An improved dataset for Fe XVII included effective collision strengths calculated by Gu (2003) with the DW approximation and the flexible atomic code (FAC, see Gu 2004). An approximate treatment of the resonances in the collision strengths was included in Gu's work. In the past few years, a few R-matrix calculations have been performed by Aggarwal et al. (2003), Chen et al. (2003), Loch et al. (2006), and more recently by Liang \& Badnell (2010) as part of the UK APAP network ${ }^{1}$.

The aim of this paper is to use these most recent R-matrix calculations and compare observed and predicted line intensities for a set of carefully selected solar observations. Results obtained from some of the previous calculations are also presented.

\footnotetext{
1 www . apap-network. org
} 
This paper is complementary to the previous paper (Del Zanna \& Ishikawa 2009), where the benchmark focused on the EUV lines of this ion. The detailed assessment of the $\mathrm{X}$-ray wavelengths, energies and identifications was published there. The present assessment work was carried out in preparation of the CHIANTI version 7 release (Landi et al. 2011). As we shall see, as in the Fe XVIII case, the main problems are finally resolved with the new R-matrix data. Section 2 briefly describes the benchmark method and atomic data used. Section 3 presents the results.

\section{The benchmark method and atomic data}

As described in Del Zanna et al. (2004), the benchmark process starts with an assessment of the observed wavelengths $\lambda_{\text {obs }}$ and a comparison with theoretical calculations, to obtain a consistent set of experimental energies for the ion. The identification of Fe XVII lines started with the excellent work of Tyrén (1938) on the soft-X-ray L-shell transitions, where identifications and very accurate wavelengths were provided. We reviewed the measurements of X-ray wavelengths found in the literature, and found that Hutcheon et al. (1976a) and Hutcheon et al. (1976b) give very accurate values. There is excellent agreement, within $1 \mathrm{~m} \AA$, between the Hutcheon et al. measurements and the most accurate measurements in the X-rays, from laboratory spectra (Boiko et al. 1978) to solar SMM/FCS ones (Phillips et al. 1982). The assessed level energies have been presented in Del Zanna \& Ishikawa (2009). In addition, the energies of the levels with $n>5$ are obtained here from the laboratory wavelengths of Brown et al. (1998).

Line intensities were calculated with the rates and transition probabilities assuming equilibrium conditions. No corrections to level populations due to ionisation/recombination effects have been included here, given that they are secondary effects. Also, the contribution of satellite lines formed by dielectronic recombination is not included here.

Line intensities were compared to observed ones to confirm identifications and assess the possible presence of blending. This was achieved using the "emissivity ratio" technique, whereby the observed intensity $I_{\mathrm{ob}}$ of a line is divided by its emissivity as a function of the electron temperature $T_{\mathrm{e}}$ :

$F_{j i}=\frac{I_{\mathrm{ob}} N_{\mathrm{e}}}{h v N_{j}\left(N_{\mathrm{e}}, T_{\mathrm{e}}\right) A_{j i}} C$

where $N_{j}\left(N_{\mathrm{e}}, T_{\mathrm{e}}\right)$ is the population of the upper level $j$ relative to the total number density of the ion, calculated at a fixed density $N_{\mathrm{e}} . A_{j i}$ is the spontaneous radiative transition probability, and $h v$ is the energy of the photon. $C$ is a scaling constant chosen so the emissivity ratio is near unity, to normalise the units of the observed intensities, and have a direct estimate of the relative agreement between observed and predicted line intensities. The same constant is chosen for each plot (set of lines).

If agreement between experimental and theoretical intensities is present, all emissivity ratios should be closely spaced. If the plasma is nearly isothermal, all curves should cross at the isothermal temperature. In one single plot, this allows one to assess which of the lines have a different sensitivity to electron temperature, and which lines are blended (see Del Zanna et al. 2004 for details).

For the present assessment we constructed four atomic models. The first adopted the excitation rates and radiative data of Liang \& Badnell (2010). The second adopted the excitation rates and radiative data of Loch et al. (2006). In both cases, the same suite of codes (see the UK APAP network website) as were originally developed for the Iron Project were used. Liang \& Badnell (2010) employed a larger target, but found very similar results to those of Loch et al. (2006). For the third atomic model we adopted the $n=3$ excitation and radiative data from Bhatia \& Doschek (1992) as included in the CHIANTI atomic package (Dere et al. 1997) version 4.2 (Young et al. 2003). Additional $n=4$ levels were present in the model. For the fourth atomic model we adopted the Gu (2003) excitation data as included in CHIANTI version 5 model (Landi et al. 2006).

The CHIANTI v.6 (Dere et al. 2009) atomic data are used for the other ions. Also, the CHIANTI v.6 ionisation equilibrium tables are adopted here. They represent a significant improvement over previous ones.

It is useful to recall that the temperature of peak ion abundance for Fe XVII in ionisation equilibrium is $\log T_{\mathrm{e}}[\mathrm{K}]=6.6$ $\left(T_{\mathrm{e}}=4 \mathrm{MK}\right)$, and that the low-density regime for this ion extends to fairly high values $\left(10^{11} \mathrm{~cm}^{-3}\right)$ that only occur in large solar flares. This means that ratios of Fe xvir lines mainly depend on electron temperature, up to high densities. This is shown in Table 1, where intensity ratios (photons) for a selection of the brightest transitions are calculated at $10^{9}, 10^{11}$, and $10^{13} \mathrm{~cm}^{-3}$ for the four atomic models and $T_{\mathrm{e}}=4 \mathrm{MK}$. The $1-52 \mathrm{p}-3 \mathrm{~s}$ $16.776 \AA$ transition is adopted as reference, following Bhatia \& Saba (2001). The table also shows the large variation in the ratio with the strongest transition (1-27 2p-3d at $15.013 \AA$ ), extensively used in the past to highlight the discrepancies with experimental data. At low densities, the ratio as predicted using the DW Bhatia \& Doschek (1992) data is 2.0, compared to a value of 1.4 using the latest R-matrix calculation (Liang \& Badnell 2010). The R-matrix data of Loch et al. (2006) provide a similar value of 1.3, while the larger model of Bhatia \& Saba (2001) improves the Bhatia \& Doschek (1992) data only by $5 \%$.

Notice that the observed 15.013/16.776 $\AA$ ratios are close to the Liang \& Badnell (2010) value. For example, the active region observation of Parkinson (1975) had a ratio (photons) of 1.39. The large discrepancy when using the DW data has been interpreted by several authors/papers as due to opacity effects in the strongest line. Although some opacity effects should be present in some conditions, it is clear that the active region observations presented in this paper suggest that no opacity effects are present.

As in the Fe XVIII case, the large differences in the different atomic calculations are caused by the resonances increasing the collision strengths of the $2 \mathrm{p}-3 \mathrm{~s}$ transitions. This is clearly shown in Fig. 1, where the collision strengths for the above lines as calculated with the R-matrix codes are displayed, together with those obtained with the DW approximation. There is overall good agreement in the background values (considering the differences in the targets), however the resonances have a large effect especially for the $2 \mathrm{p}-3 \mathrm{~s}$ transition. The presence of the resonances substantially increases the thermally-averaged collision strengths, as displayed in Fig. 2.

\section{X-ray observations}

Fe xvII emission is normally produced on the Sun all the time by every quiescent active region in its core. That this hot emission is nearly isothermal around $3 \mathrm{MK}$ has been suggested for a long time. For example, Rosner et al. (1978) inferred this from broad-band X-ray Skylab images. Many SMM observations also suggested this characteristic (see, e.g. Schmelz et al. 1996), later confirmed by SOHO/CDS (Del Zanna \& Mason 2003). The quiescent hot emission therefore represent an excellent case to 
Table 1. List of main Fe xvII X-ray lines and intensity ratios.

\begin{tabular}{|c|c|c|c|c|c|c|c|c|}
\hline Key & $I_{\mathrm{ob}}$ & $i-j$ & Terms & $\begin{array}{c}I \\
10^{9}\end{array}$ & $\begin{array}{c}I \\
10^{11}\end{array}$ & $\begin{array}{c}I \\
10^{13}\end{array}$ & $\lambda_{\exp }(\AA)$ & \\
\hline \multirow[t]{5}{*}{4} & 111.26 & $1-27$ & $2 s^{2} 2 p^{6}{ }^{1} S_{0}-2 p^{5} 3 d^{1} P_{1}$ & 1.4 & 1.4 & 1.2 & 15.013 & LB10 \\
\hline & & & & 1.3 & 1.3 & 1.2 & & L06 \\
\hline & & & & 2.0 & 2.0 & 1.9 & & BD92 \\
\hline & & & & 1.9 & 1.89 & 1.79 & & BS01 \\
\hline & & & & 1.5 & 1.5 & 1.4 & & G03 \\
\hline \multirow[t]{5}{*}{8} & 103.68 & $1-3$ & $2 \mathrm{~s}^{2} 2 \mathrm{p}^{6}{ }^{1} \mathrm{~S}_{0}-2 \mathrm{p}^{5} 3 \mathrm{~s}^{1} \mathrm{P}_{1}$ & 1.3 & 1.3 & 1.1 & 17.051 & LB10 \\
\hline & & & & 1.3 & 1.3 & 1.1 & & L06 \\
\hline & & & & 1.1 & 1.1 & 1.0 & & BD92 \\
\hline & & & & 1.17 & 1.17 & 1.09 & & BS01 \\
\hline & & & & 1.3 & 1.2 & 1.1 & & G03 \\
\hline \multirow[t]{5}{*}{9} & 81.78 & $1-2$ & $2 \mathrm{~s}^{2} 2 \mathrm{p}^{6}{ }^{1} \mathrm{~S}_{0}-2 \mathrm{p}^{5} 3 \mathrm{~s}^{3} \mathrm{P}_{2}$ & 1.1 & 1.1 & 0.94 & 17.096 & LB10 \\
\hline & & & & 1.0 & 1.0 & 0.87 & & L06 \\
\hline & & & & 0.84 & 0.84 & 0.69 & & BD92 \\
\hline & & & & 0.81 & 0.81 & 0.71 & & BS01 \\
\hline & & & & 0.98 & 0.98 & 0.82 & & G03 \\
\hline 7 & 71.6 & $1-5$ & $2 s^{2} 2 p^{6}{ }^{1} S_{0}-2 p^{5} 3 s^{3} P_{1}$ & 1.0 & 1.0 & 1.0 & 16.776 & \\
\hline \multirow[t]{5}{*}{5} & 53.32 & $1-23$ & $2 s^{2} 2 p^{6}{ }^{1} S_{0}-2 p^{5} 3 d^{3} D_{1}$ & 0.40 & 0.40 & 0.36 & 15.262 & LB10 \\
\hline & & & & 0.42 & 0.42 & 0.37 & & L06 \\
\hline & & & & 0.49 & 0.49 & 0.45 & & BD92 \\
\hline & & & & 0.51 & 0.51 & 0.48 & & BS01 \\
\hline & & & & 0.44 & 0.44 & 0.40 & & G03 \\
\hline 1 & 9.66 & $1-71$ & $2 \mathrm{~s}^{2} 2 \mathrm{p}^{6}{ }^{1} \mathrm{~S}_{0}-2 \mathrm{p}^{5} 4 \mathrm{~d}^{1} \mathrm{P}_{1}$ & 0.12 & 0.12 & 0.11 & 12.124 & LB10 \\
\hline 2 & 8.84 & $1-59$ & $2 s^{2} 2 p^{6}{ }^{1} S_{0}-2 p^{5} 4 d^{3} D_{1}$ & 0.11 & 0.11 & $9.9 \times 10^{-2}$ & 12.264 & LB 10 \\
\hline 3 & 8.12 & $1-33$ & $2 \mathrm{~s}^{2} 2 \mathrm{p}^{6}{ }^{1} \mathrm{~S}_{0}-2 \mathrm{p}^{6} 3 \mathrm{p}^{1} \mathrm{P}_{1}$ & $9.7 \times 10^{-2}$ & $9.6 \times 10^{-2}$ & $8.7 \times 10^{-2}$ & 13.825 & LB10 \\
\hline \multirow[t]{19}{*}{6} & 12.82 & $1-17$ & $2 \mathrm{~s}^{2} 2 \mathrm{p}^{6}{ }^{1} \mathrm{~S}_{0}-2 \mathrm{p}^{5} 3 \mathrm{~d}^{3} \mathrm{P}_{1}$ & $8.4 \times 10^{-2}$ & $8.4 \times 10^{-2}$ & $7.8 \times 10^{-2}$ & 15.453 & LB10 \\
\hline & & & & $8.0 \times 10^{-2}$ & $8.0 \times 10^{-2}$ & $7.4 \times 10^{-2}$ & & L06 \\
\hline & & & & $8.6 \times 10^{-2}$ & $8.6 \times 10^{-2}$ & $8.2 \times 10^{-2}$ & & BD92 \\
\hline & & & & $8.69 \times 10^{-2}$ & $8.67 \times 10^{-2}$ & $8.42 \times 10^{-2}$ & & BS01 \\
\hline & & & & $8.1 \times 10^{-2}$ & $8.1 \times 10^{-2}$ & $7.5 \times 10^{-2}$ & & G03 \\
\hline & 3.9 & $1-93$ & $2 \mathrm{~s}^{2} 2 \mathrm{p}^{6}{ }^{1} \mathrm{~S}_{0}-2 \mathrm{p}^{5} 5 \mathrm{~d}^{1} \mathrm{P}_{1}$ & $4.3 \times 10^{-2}$ & $4.3 \times 10^{-2}$ & $3.9 \times 10^{-2}$ & 11.250 & LB10 \\
\hline & 3.3 & $1-118$ & $2 s^{2} 2 p^{6}{ }^{1} S_{0}-2 p^{5} 5 d^{3} D_{1}$ & $3.1 \times 10^{-2}$ & $3.1 \times 10^{-2}$ & $2.8 \times 10^{-2}$ & 11.129 & LB10 \\
\hline & 3.18 & $1-7$ & $2 s^{2} 2 p^{6}{ }^{1} S_{0}-2 p^{5} 3 p^{3} D_{2}$ & $2.8 \times 10^{-2}$ & $2.8 \times 10^{-2}$ & $3.1 \times 10^{-2}$ & 16.336 & LB10 \\
\hline & & & & $2.5 \times 10^{-2}$ & $2.5 \times 10^{-2}$ & $2.8 \times 10^{-2}$ & & L06 \\
\hline & & & & $2.3 \times 10^{-2}$ & $2.3 \times 10^{-2}$ & $2.8 \times 10^{-2}$ & & BD92 \\
\hline & & & & $2.2 \times 10^{-2}$ & $2.2 \times 10^{-2}$ & $2.56 \times 10^{-2}$ & & BS01 \\
\hline & & & & $2.4 \times 10^{-2}$ & $2.4 \times 10^{-2}$ & $2.8 \times 10^{-2}$ & & G03 \\
\hline & 4.44 & $1-31$ & $2 s^{2} 2 p^{6}{ }^{1} S_{0}-2 p^{6} 3 p^{3} P_{1}$ & $2.3 \times 10^{-2}$ & $2.3 \times 10^{-2}$ & $2.1 \times 10^{-2}$ & 13.890 & LB10 \\
\hline & 26.6 & $1-14$ & $2 s^{2} 2 p^{6}{ }^{1} S_{0}-2 p^{5} 3 p^{1} D_{2}$ & $2.3 \times 10^{-2}$ & $2.3 \times 10^{-2}$ & $2.1 \times 10^{-2}$ & 16.004 & (bl O VIII) LB10 \\
\hline & 2.62 & $1-155$ & $2 s^{2} 2 p^{6}{ }^{1} S_{0}-2 p^{5} 6 d^{1} P_{1}$ & $2.2 \times 10^{-2}$ & $2.2 \times 10^{-2}$ & $1.9 \times 10^{-2}$ & 10.770 & LB10 \\
\hline & 1.26 & $1-165$ & $2 s^{2} 2 p^{6}{ }^{1} S_{0}-2 p^{5} 6 d^{3} D_{1}$ & $1.2 \times 10^{-2}$ & $1.2 \times 10^{-2}$ & $1.1 \times 10^{-2}$ & 10.657 & LB 10 \\
\hline & 3.04 & $1-39$ & $2 s^{2} 2 p^{6}{ }^{1} S_{0}-2 p^{5} 4 s^{1} P_{1}$ & $1.1 \times 10^{-2}$ & $1.1 \times 10^{-2}$ & $9.7 \times 10^{-3}$ & 12.680 & LB 10 \\
\hline & 1.54 & $1-131$ & $2 s^{2} 2 p^{6}{ }^{1} S_{0}-2 p^{6} 4 p^{1} P_{1}$ & $7.2 \times 10^{-3}$ & $7.2 \times 10^{-3}$ & $6.5 \times 10^{-3}$ & 11.023 & LB10 \\
\hline & 1.04 & $1-197$ & $2 s^{2} 2 p^{6}{ }^{1} S_{0}-2 p^{6} 5 p^{1} P_{1}$ & $1.8 \times 10^{-3}$ & $1.8 \times 10^{-3}$ & $1.6 \times 10^{-3}$ & 10.115 & LB 10 \\
\hline
\end{tabular}

Notes. The first column gives the key numbers for Fig. 4. The second column $\left(I_{\mathrm{ob}}\right)$ provides the Parkinson (1975) observed intensity, in $10^{-6} \mathrm{erg} \mathrm{cm}^{-2} \mathrm{~s}^{-1}$. The third and fourth columns provide the indices and description for the transitions. The following three columns give the intensity ratios (photons) calculated at $10^{9}, 10^{11}$, and $10^{13} \mathrm{~cm}^{-3}$ and $4 \mathrm{MK}$, from the atomic data of: LB10: Liang \& Badnell (2010); L06: Loch et al. (2006); BD92: data from CHIANTI version 4.2, which included the DW effective collision strengths calculated by Bhatia \& Doschek (1992); BS01: data from the larger model of Bhatia \& Saba (2001); G03: data from CHIANTI version 5.2, which included effective collision strengths calculated by $\mathrm{Gu}$ (2003). $\lambda_{\text {exp }}$ is the experimental wavelength.

apply the emissivity ratio technique. We have applied the technique to an extensive range of observations. Only some selected results are shown here as examples. We start with the excellent X-ray spectrum of a quiescent active region described by Parkinson (1975). The instrument, built by the University of Leicester (UK), consisted of Bragg crystal spectrometers with a collimator having a FOV (FWHM) of $3^{\prime}$, and flown on a British Skylark sounding rocket on 30 Nov. 1971.

We consider Parkinson's spectrum to be the best X-ray spectrum of a quiescent active region for a variety of reasons. To start with, there are very few such observations, most of them either being of the full Sun or of flares. Parkinson's instrument had an excellent spectral resolution and observed a large number of weak lines. Indeed many lines were identified by Parkinson for the first time. The instrument was radiometrically calibrated and covered at once a wide spectral region. The observations obtained with the KAP crystal in the 9-23 $\AA$ range are used here. The Fe XVII lines were prominent, while the strongest Fe XVIII 14.20 A self-blend was barely visible. Lines formed at higher temperatures were totally absent, indicating a lack of plasma with $T>4 \mathrm{MK}$.

One simple way to estimate if the plasma is nearly isothermal is the EM loci method, by which the curves $I_{\mathrm{ob}} /(A \times G(T))$ as a function of temperature are plotted $\left(I_{\mathrm{ob}}\right.$ is the observed intensity, 

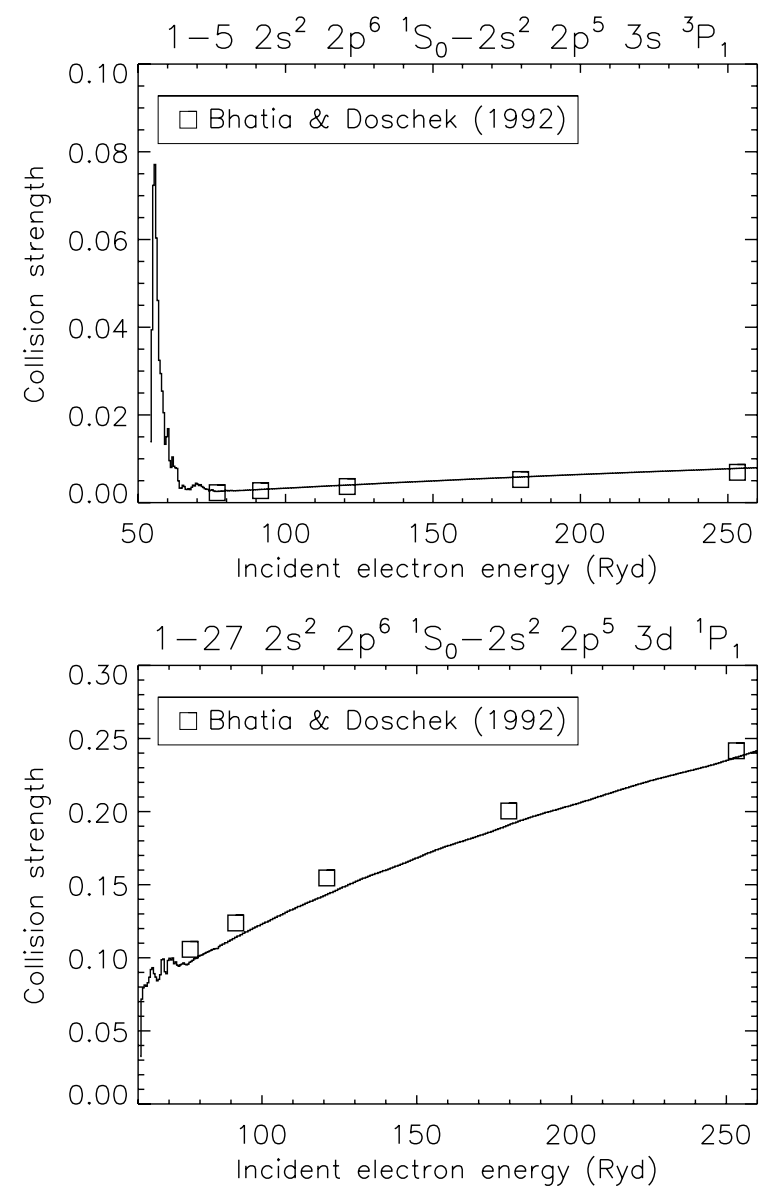

Fig. 1. The collision strengths for two of the main Fe xVII lines. The R-matrix data of Loch et al. (2006), averaged for displaying purposes, are shown together with the DW data of Bhatia \& Doschek (1992).

$A$ the element abundance and $G(T)$ the contribution function containing the atomic parameters). If the curves cross at one temperature, then the plasma is most likely isothermal. For details, see Strong (1978) and Del Zanna et al. (2002).

The EM loci curves for a few strong lines are shown in Fig. 3 and confirm that the plasma is nearly isothermal with $T=3 \mathrm{MK}$ $(\log T[\mathrm{~K}]=6.5)$. On a side note, it is interesting to point out that the relative $\mathrm{Ne} / \mathrm{Fe}$ abundance adopted for the EM loci plot is 1.34 , i.e. half of the value suggested by the latest compilation of "photospheric" abundances (Asplund et al. 2009, although the $\mathrm{Ne}$ abundance was actually inferred from coronal observations). In other words, the first ionisation potential (FIP) bias in this case is two.

Figure 4 shows the emissivity ratio curves of the strongest and most used lines, for the Parkinson (1975) active region spectrum. Each curve is numbered, and the details are provided in Table 1. With the notable exception of the 15.26 and $15.45 \AA$ lines (numbers 5 and 6 ), excellent (to within a relative $\pm 10 \%$ ) agreement is found around $\log T[\mathrm{~K}]=6.5$ with the Liang \& Badnell (2010) atomic data. The curves also indicate that the ratio of any of the strong $2 p-3 d$ with any of the strong $2 p-3$ s transitions is a good temperature diagnostic, as found for Fe xvIII by Del Zanna (2006). The 2p-4d and 2p-3p lines (numbers 1, 2, and 3 ) can also provide temperature measurements when used in conjunction with the $2 \mathrm{p}-3 \mathrm{~d}$ lines.

Almost the same results are obtained with the Loch et al. (2006) calculations. This is because, as shown in Liang \& Badnell (2010), the actual collisional and radiative rates for
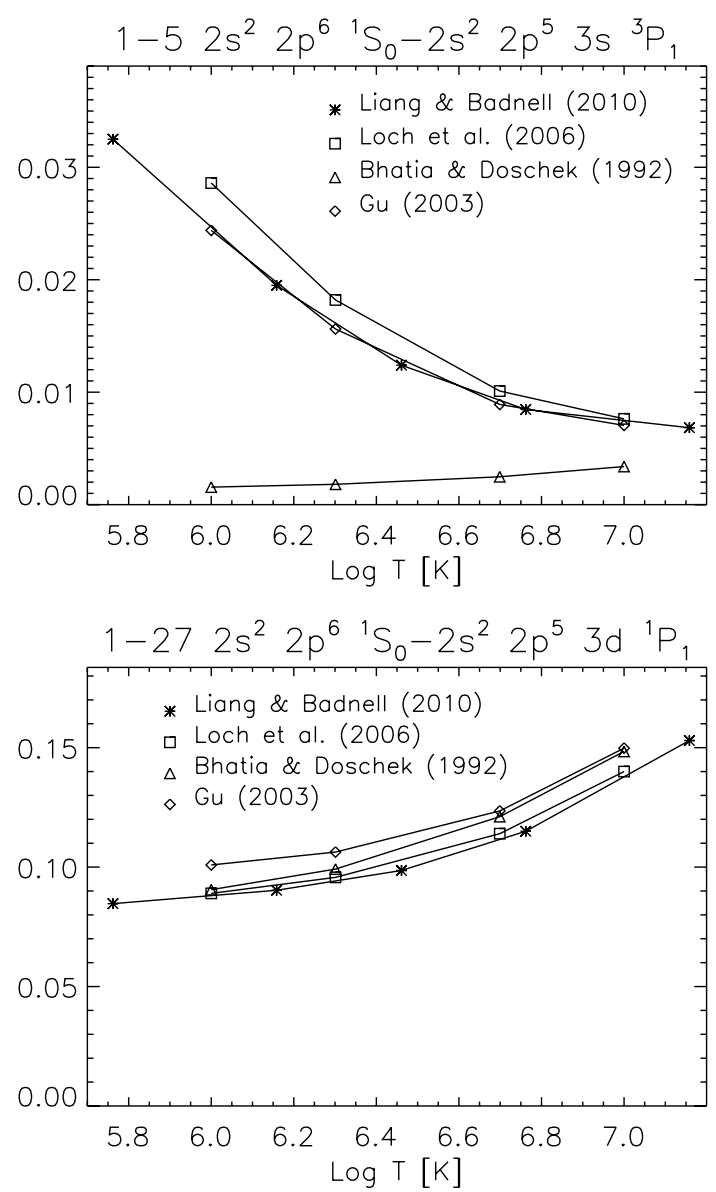

Fig. 2. The thermally-averaged collision strengths for two of the main Fe XVII lines.

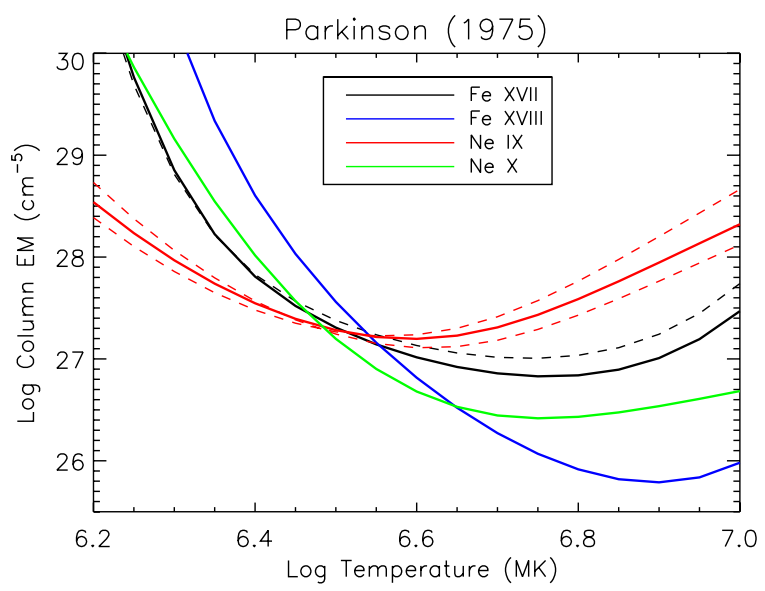

Fig. 3. The EM loci plot for the (Parkinson 1975) active region spectrum. The continuous lines are for the resonance lines: Fe XVII $15.01 \AA$, Fe xvIII 14.20 A self-blend, the He-like Ne Ix $13.45 \AA\left(1 \mathrm{~s}^{2}-1 \mathrm{~s} 2 \mathrm{p}\right)$, and the H-like self-blend $\mathrm{Ne} x 12.1 \AA$ (1s-2p). The dashed lines are for the Fe XVII $17.05 \AA$, Ne Ix $13.70 \AA$ (intercombination) and $11.55 \AA\left(1 \mathrm{~s}^{2}-1 \mathrm{~s}\right.$ $3 p)$.

the strongest lines are almost the same. The well-known large discrepancies when using the DW calculations of Bhatia \& Doschek (1992) are also evident in Fig. 4. The same figure also shows that the atomic data of $\mathrm{Gu}$ (2003) provide a remarkable agreement with observations. 
G. Del Zanna: Benchmarking atomic data for astrophysics: Fe xviI X-ray lines
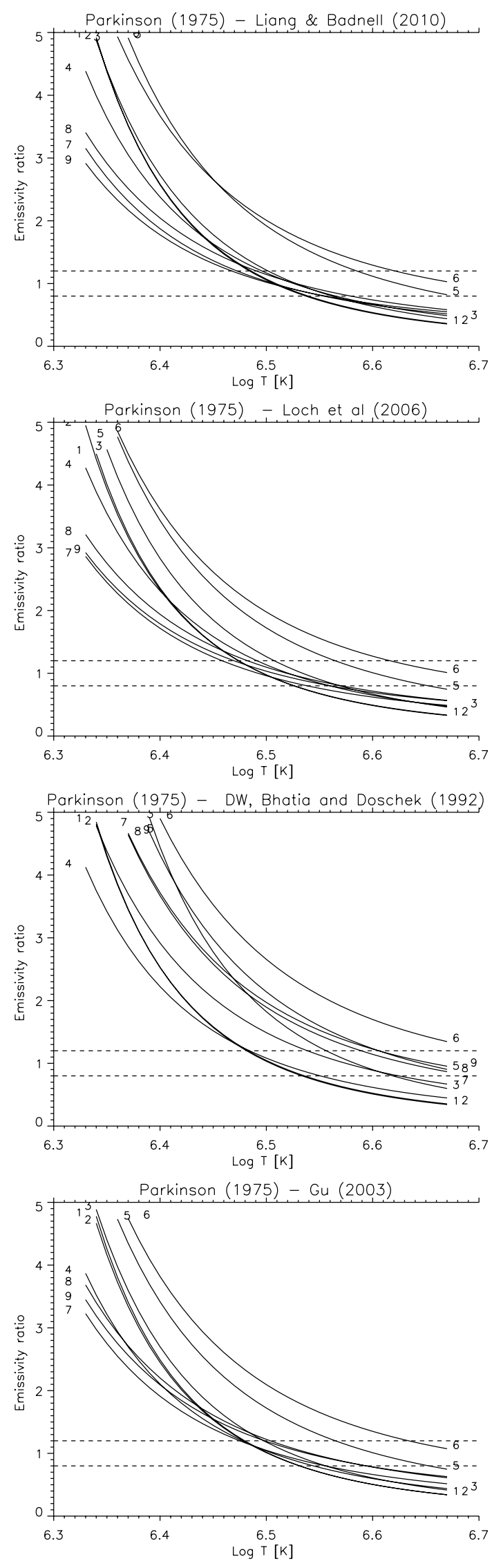

Fig. 4. Emissivity ratio curves of the strongest and most used lines in the (Parkinson 1975) active region spectrum, obtained with four different atomic datasets. The curves are numbered (see Table 1 for details). The dashed lines are plotted at $\pm 20 \%$.

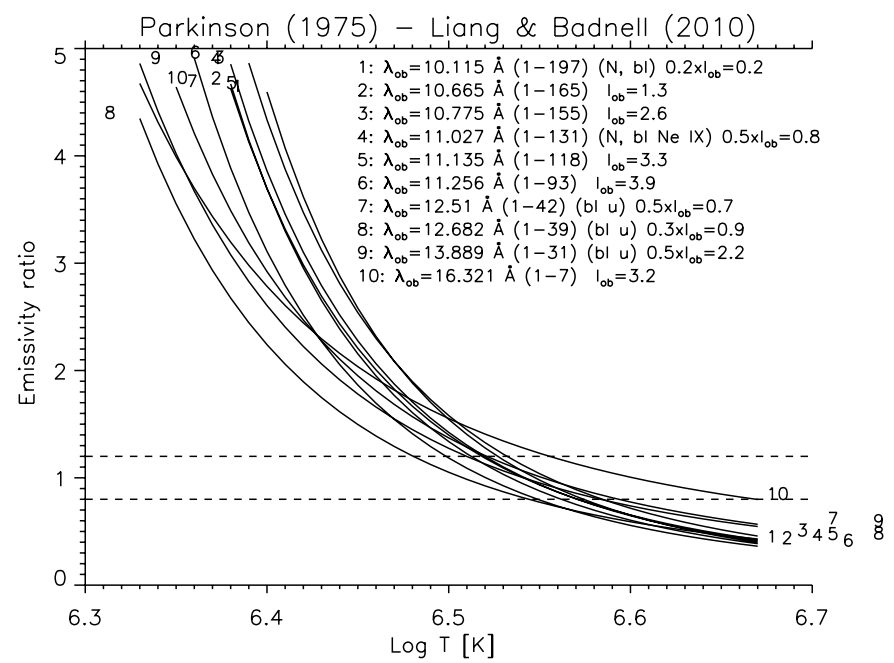

Fig. 5. Emissivity ratio curves of the weaker lines in the Parkinson (1975) active region spectrum. $\lambda_{\mathrm{ob}}$ indicates the observed wavelength. The indices the lower and upper level are then shown. $I_{\mathrm{ob}}$ indicates the observed intensity, in $10^{-6} \mathrm{erg} \mathrm{cm}^{-2} \mathrm{~s}^{-1}$. "N" is a new identification. "bl" indicates the presence of a blend, "u" with an unidentified line. The observed intensities of a few lines have been multiplied by the factors shown.

Figure 5 shows the emissivity ratio curves of the weaker lines. Most (but not all) were identified by Parkinson. Many of them are however obviously blended, and some additional identifications are provided here. The intensities of some of the blended lines have been reduced as indicated in the figure.

Line no.1 (1-197) is a tentative new identification of one line of the $2 p-5 p$ transition array. The difference between observed and theoretical energies of nearby levels (e.g., $2 \mathrm{p}^{5} 7 \mathrm{~d}$ ) is about $12000 \mathrm{~cm}^{-1}$. By applying a similar correction to the $5 \mathrm{p}$ levels, the wavelength of the 1-197 transition is then predicted to fall at $10.11 \AA$. The intensities of the $2 \mathrm{p}-6 \mathrm{~d}$ and $2 \mathrm{p}-5 \mathrm{~d}$ lines identified by Parkinson are in excellent agreement with the predicted ones. The $11.02 \AA$ line was identified by Parkinson as being due to Ne Ix, however about $50 \%$ of its intensity is due to the Fe XVII 1131 line. The $2 p-4 s$ lines and the $2 p-3 p$ 1-31 line are obviously blended.

The 1-7 transition is predicted to fall at $16.34 \AA$ adopting the SERTS-89 (Thomas \& Neupert 1994) EUV measurement at $367.287 \AA$ of the $2-7$ transition (see Del Zanna \& Ishikawa 2009). This is in excellent agreement with the wavelength of a line observed with the SMM/FCS at $16.337 \AA$ (Phillips et al. 1982). Brown et al. (1998) observed the line at $16.350 \pm 0.008 \AA$, so it appears that this measurement is inaccurate. There is a line left unidentified by Parkinson and observed at $16.321 \AA$, with almost the expected intensity of the 1-7 line, so it seems likely that the Parkinson wavelength was also not correct. Indeed various lines in the Parkinson list have wavelengths that differ from other measurements.

After the Skylark rocket spectrum, the best X-ray spectra have been obtained with the SMM/FCS. The SMM database ${ }^{2}$ was searched for quiescent AR observations (with little hot emission), following the long lists of selected FCS data by Schmelz et al. (1996) and Phillips et al. (1997). The SMM/FCS observations considered here started with a monochromatic image of the active region. The instrument was then pointed at the brightest pixel of that image and scanned the 13-20 A region. Each scan lasted about $10 \mathrm{~min}$.

2 ftp://umbra.nascom.nasa.gov/pub/smm/xrp/data 


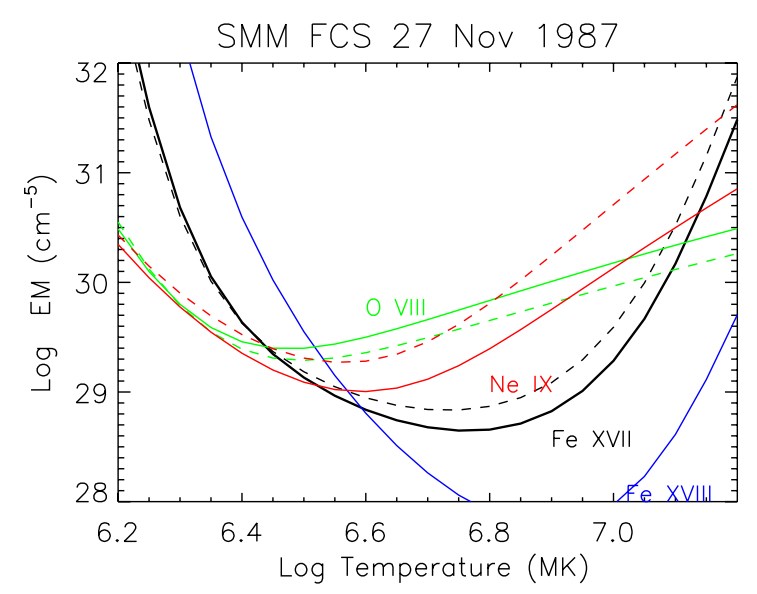

Fig. 6. The EM loci plot for the SMM/FCS AR observation on 27 Nov. 1987. The continuous lines are for the resonance lines: Fe XVII 15.01 $\AA$, Fe xVIII $14.20 \AA$ self-blend, O VIII $18.97 \AA$ self-blend, Ne Ix $13.45 \AA$. The dashed lines are for the Fe xvII 17.05 $\AA$, O VIII $16.00 \AA$, Ne Ix $13.70 \AA$.

We looked at the count rates in the CaxvIII/Ca XIX channel of the SMM bent crystal spectrometer (BCS) during each scanning, to look for observations where count rates were stationary. This is necessary when comparing intensities of lines observed at different times (wavelengths). Many observations were found not suitable, however a few were, and have been analysed here. Only the results from the observations of 27 Nov. 1987 on AR 4891 are presented. To increase the signal-to-noise, three spectral scans, taken at 16:25, 16:35 and 16:45 UT were averaged. The spectra have been flux-calibrated using the current software available within Solarsoft ${ }^{3}$.

The spectral lines were fitted with Voigt profiles with custom-written software to obtain line intensities. The FCS collimator angular (FWHM) dimensions are approximately 15" (equivalent to $225 \operatorname{arcsec}^{2}$ ), but are slightly dependent on the wavelength (Phillips, priv. comm.). Intensities have been converted to phot $\mathrm{cm}^{-2} \mathrm{~s}^{-1} \operatorname{arcsec}^{-2}$.

As in many other SMM/FCS observations, the FexvIII $14.20 \AA$ self-blend is barely above noise, so its intensity is very uncertain (about 50\%). This in itself is an interesting observation confirming, as in the Parkinson (1975) case, the lack of hot emission above $4 \mathrm{MK}$ in the cores of active regions. This topic is currently debated, given its implications for the modelling of the heating via nanoflares (see, e.g. Patsourakos \& Klimchuk 2009).

Figure 6 shows the EM loci curves for a selection of lines. The crossing of the Fe XVIII 14.20 A self-blend with the Fe XVII lines indicates a temperature at or below $\log T[\mathrm{~K}]=6.6$. The $\mathrm{O}$ VIII and $\mathrm{Ne}$ IX lines indicate instead a temperature closer to $\log T[\mathrm{~K}]=6.4$, similar to the Fe XVII measurement. In order to match the emission measures, the oxygen/iron and neon/iron abundances were reduced by a factor of four, compared to the (latest) photospheric abundances of Asplund et al. (2009), where they are 15.5 and 2.69 respectively. This results in $\mathrm{O} / \mathrm{Fe}$ and $\mathrm{Ne} / \mathrm{Fe}$ relative abundances of 3.87 and 0.67 . On a side note, it is interesting to compare the Schmelz et al. (1996) results obtained from the same observation. They considered as "standard" photospheric abundances for $\mathrm{O} / \mathrm{Fe}$ and $\mathrm{Ne} / \mathrm{Fe}$ values of 5.0 and 0.76 , respectively. The observation is listed as having low $\mathrm{Ne}$ and $\mathrm{O}$ abundances, however the results here are, within uncertainties, close to their "standard" values. In general, differences with the Schmelz et al. (1996) results have been found (details will

\footnotetext{
3 www.lmsal.com/solarsoft/
}

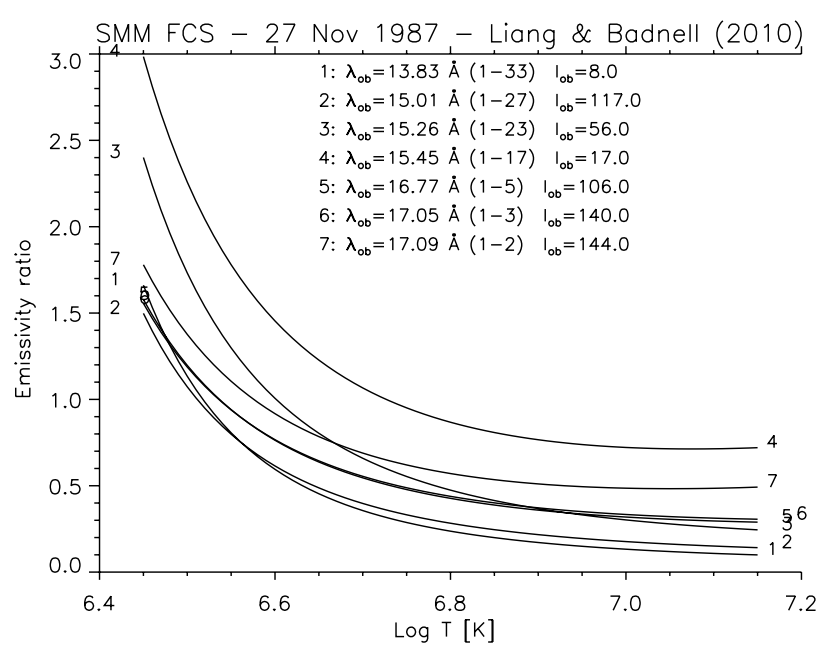

Fig. 7. The emissivity ratio curves relative to the $3 \rightarrow 2$ transitions observed in AR by the SMM/FCS on 27 Nov. 1987, with the Liang \& Badnell (2010) atomic data. $I_{\mathrm{ob}}$ is the measured observed intensity, in phot $\mathrm{cm}^{-2} \mathrm{~s}^{-1} \operatorname{arcsec}^{-2}$.

appear in a forthcoming paper). These are most likely due to the different atomic data and the different ion equilibrium abundances adopted here.

Figure 7 shows the emissivity ratio curves obtained from the SMM/FCS observation and the Liang \& Badnell (2010) atomic data. With the exception of the weaker $15.26,15.45 \AA$ lines, excellent (to within a relative $10 \%$ ) agreement between observed and predicted intensities is found, for temperatures around $\log$ $T[\mathrm{~K}]=6.5$ (as also indicated by the EM loci plot), although lower temperatures cannot be ruled out. The weaker $2 \mathrm{p}-3 \mathrm{p}$ 13.83 A line, not included in Phillips et al. (1997), also shows excellent agreement. Very similar curves are obtained with the Loch et al. (2006) data, while the DW Bhatia \& Doschek (1992) data present the same level of disagreement as seen for the Parkinson's spectrum. The other SMM FCS AR observations analysed here provide very similar results.

In order to benchmark the atomic data against higher- $T$ plasma, many datasets have been considered. The obvious candidate is a solar flare. There are plenty of such observations, however the main drawback in many cases was that data were obtained with crystal spectrometers, and it took considerable time to scan a wavelength region. During the impulsive phase of a flare, line intensities can vary by orders of magnitude, so the gradual phase is to be preferred in those cases. A second drawback is the fact that the emission of the flaring plasma is not necessarily isothermal. Very large flares are likely to have multithermal plasmas in the line of sight, however small (e.g. B- or C-class) flares have been shown to be close to isothermal at their peak and during the cooling phase (see, e.g. Del Zanna et al. 2011).

An exception to the first drawback is the high-quality solar spectrum (Acton et al. 1985) recorded on photographic film with an exposure of $145 \mathrm{~s}$ during a rocket flight, 2 min after the GOES $\mathrm{X}$-ray peak emission of an M1-class flare. Figure 8 shows the corresponding emissivity ratios with the Liang \& Badnell (2010) atomic data. Excellent (to within a relative 10\%) agreement between observed and predicted intensities is found for temperatures around $\log T[\mathrm{~K}]=7$, with three exceptions: the 17.10 and $15.45 \AA$ lines are brighter than predicted by about $20-30 \%$. This could be due to blending. The resonance $15.0 \AA$ line has a weaker observed intensity. This could be caused by opacity 


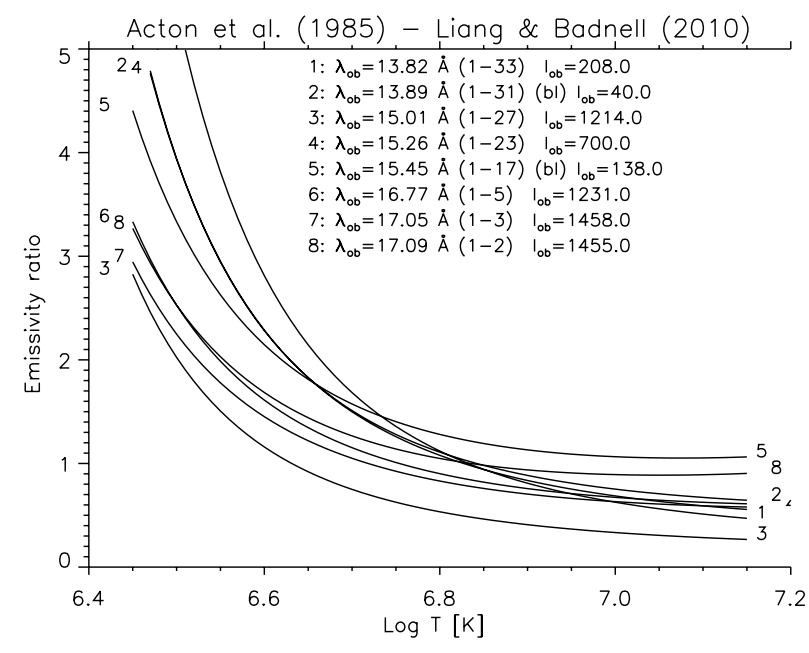

Fig. 8. The emissivity ratio curves of the M1-class flare of the $13 \mathrm{Jul}$. 1982 (Acton et al. 1985) and the Liang \& Badnell (2010) atomic data. $I_{\mathrm{ob}}$ is the measured observed intensity, in phot $\mathrm{cm}^{-2} \mathrm{~s}^{-1} \operatorname{arcsec}^{-2}$.

effects, given the high densities during the flare, or by saturation effects.

The SMM/BCS observations in the Ca XvII/Ca XIX channel have been searched to find a reasonably large flare with a slowlydecreasing gradual phase. Results are presented from the analysis of the M5-class flare of 19 Oct. 1986 and the FCS scan that started at 01:49 UT. During this scan, the Ca XVIII/Ca XIX count rates decreased by only about $20 \%$, i.e. any solar variability effects would be at most of this order. The observation has been processed and showed a richness of emission lines.

Figure 9 shows the emissivity ratio curves obtained from the Fe XVIII and Fe XVII lines present in the spectrum. For Fe XVIII, the atomic data discussed in Witthoeft et al. (2006) and Del Zanna (2006) were adopted. For Fe xvII, the Liang \& Badnell (2010) atomic data were used. For both ions, good (to within a relative $20 \%$ ) agreement is found for $\log T[\mathrm{~K}]=6.65$, with the exception of the Fe XVII 15.26 and $17.10 \AA$ lines, which are slightly offset.

\section{Conclusions}

As was the case for the Fe XvIII lines (Del Zanna 2006), the recent R-matrix scattering calculations finally resolve longstanding discrepancies between observed and predicted line intensities for Fe xvII. Excellent agreement (to within a relative $\pm 10 \%$ ) is found between observed and predicted line intensities from a selection of carefully chosen solar observations, and the Liang \& Badnell (2010) (or Loch et al. 2006) atomic data.

The controversial presence of opacity effects in solar active regions is ruled out by the new atomic data, although some effects appear to be present during the peak phase of large solar flares.

The new atomic data, combined with the most recent ionisation equilibrium tables, provide emission measures that are significantly different than previous ones, affecting measurements of relative elemental abundances. $\mathrm{O} / \mathrm{Fe}$ and $\mathrm{Ne} / \mathrm{Fe}$ abundances are found in quiecent active regions reduced by factors of two to four compared to the latest compilation of "photospheric" abundances.

We have clearly shown for the first time that, together with Fe XVIII, the strongest Fe XVII lines can now be reliably used for the measurement of electron temperatures in the solar corona
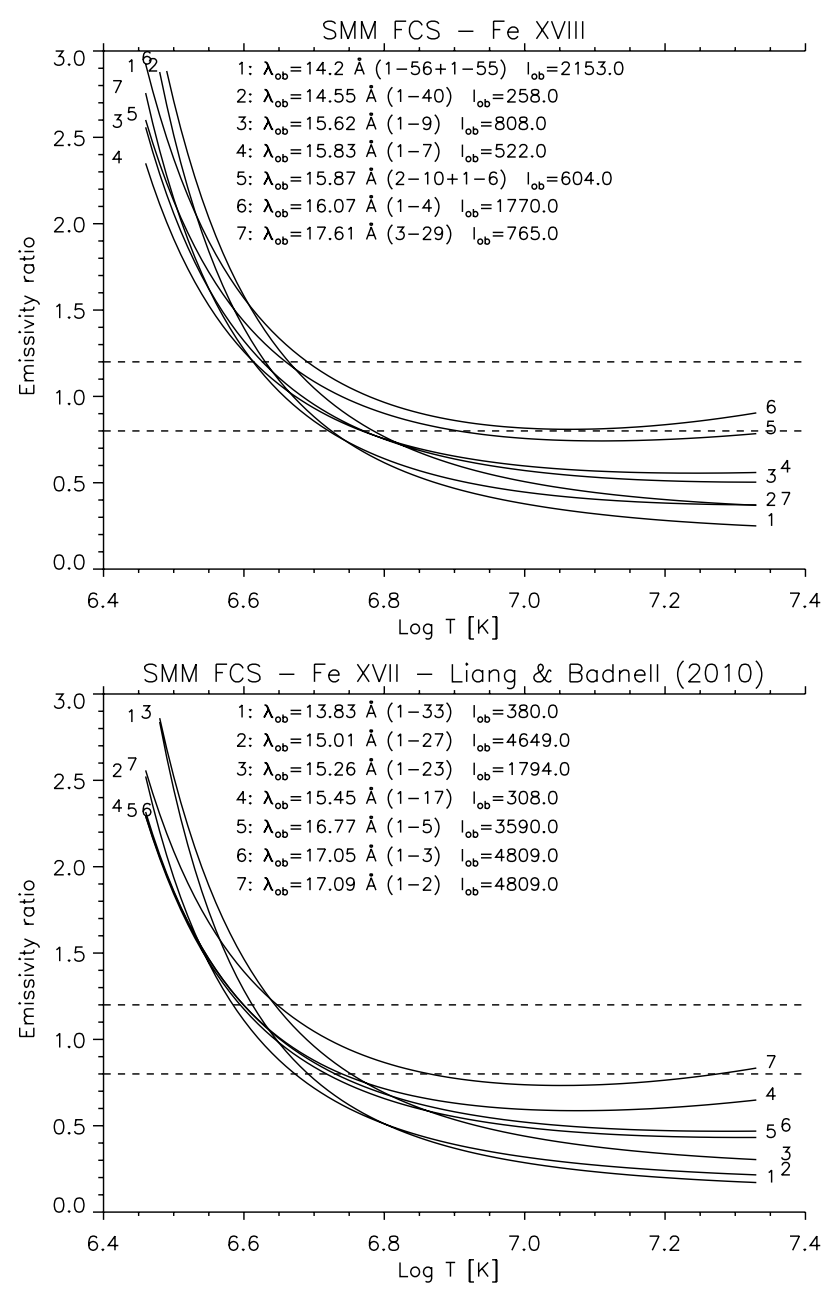

Fig. 9. The emissivity ratio curves relative to the flare of 19 Oct. 1986 observed by SMM/FCS, for Fe XVIII (above) and Fe XVII (below) lines. $I_{\mathrm{ob}}$ is the measured observed intensity, in phot $\mathrm{cm}^{-2} \mathrm{~s}^{-1} \operatorname{arcsec}^{-2}$.

and other astrophysical sources. The new atomic model has allowed the tentative identifications of a few lines and the presence of a few blends for some of the weaker lines that were not known before. More work is in progress to assess the entire atomic data for the X-rays, resolve the outstanding blending issues, and revise previous measurements of chemical abundances.

Acknowledgements. Support from STFC (UK) via the Advanced Fellowship and the APAP network is acknowledged. C. Ballance is thanked for providing the Loch et al. atomic data. All the people that helped in locating the SMM data are thanked, in particular: J. Gurman, L. Acton, S. Freeland, K. Strong, J. Saba, L. Culhane. K. Phillips is thanked for useful comments on the SMM FCS collimator. The anonymous referee is thanked for the very constructive comments.

\section{References}

Acton, L. W., Bruner, M. E., Brown, W. A., et al. 1985, ApJ, 291, 865 Aggarwal, K. M., Keenan, F. P., \& Msezane, A. Z. 2003, ApJS, 144, 169 Asplund, M., Grevesse, N., Sauval, A. J., \& Scott, P. 2009, ARA\&A, 47, 481 Bhatia, A. K., \& Doschek, G. A. 1992, Atomic Data and Nuclear Data Tables, 52,1

Bhatia, A. K., \& Saba, J. L. R. 2001, ApJ, 563, 434

Boiko, V. A., Faenov, A. I., \& Pikuz, S. A. 1978, J. Quant. Spectros. Rad. Trans., 19,11

Brown, G. V., Beiersdorfer, P., Liedahl, D. A., Widmann, K., \& Kahn, S. M. 1998, ApJ, 502, 1015

Chen, G.-X., Pradhan, A. K., \& Eissner, W. 2003, J. Phys. B Atom. Mol. Phys., 36,453 
Del Zanna, G. 2006, A\&A, 459, 307

Del Zanna, G., \& Mason, H. E. 2003, A\&A, 406, 1089

Del Zanna, G., \& Ishikawa, Y. 2009, A\&A, 508, 1517

Del Zanna, G., Landini, M., \& Mason, H. E. 2002, A\&A, 385, 968

Del Zanna, G., Berrington, K. A., \& Mason, H. E. 2004, A\&A, 422, 731

Del Zanna, G., Mitra-Kraev, U., Bradshaw, S. J., Mason, H. E., \& Asai, A. 2011, A\&A, 526, A1

Dere, K. P., Landi, E., Mason, H. E., Monsignori Fossi, B. C., \& Young, P. R. 1997, A\&AS, 125, 149

Dere, K. P., Landi, E., Young, P. R., et al. 2009, A\&A, 498, 915

Gu, M. F. 2003, ApJ, 582, 1241

Gu, M. F. 2004, in AIP Conf. Ser. 730, ed. J. S. Cohen, D. P. Kilcrease, \& S. Mazavet, 127

Hutcheon, R. J., Pye, J. P., \& Evans, K. D. 1976a, MNRAS, 175, 489

Hutcheon, R. J., Pye, J. P., \& Evans, K. D. 1976b, A\&A, 51, 451

Landi, E., Del Zanna, G., Young, P. R., et al. 2006, ApJS, 162, 261

Landi, E., Del Zanna, G., Young, P. R., Dere, K. P., \& Mason, H. E. 2011, ApJS, in press
Liang, G. Y., \& Badnell, N. R. 2010, A\&A, 518, A64

Loch, S. D., Pindzola, M. S., Ballance, C. P., \& Griffin, D. C. 2006, J. Phys. B Atom. Mol. Phys., 39, 85

Parkinson, J. H. 1975, Sol. Phys., 42, 183

Patsourakos, S., \& Klimchuk, J. A. 2009, ApJ, 696, 760

Phillips, K. J. H., Fawcett, B. C., Kent, B. J., et al. 1982, ApJ, 256, 774

Phillips, K. J. H., Greer, C. J., Bhatia, A. K., \& Keenan, F. P. 1996, ApJ, 469, L57

Phillips, K. J. H., Greer, C. J., Bhatia, A. K., et al. 1997, A\&A, 324, 381

Rosner, R., Tucker, W. H., \& Vaiana, G. S. 1978, ApJ, 220, 643

Saba, J. L. R., Schmelz, J. T., Bhatia, A. K., \& Strong, K. T. 1999, ApJ, 510, 1064

Schmelz, J. T., Saba, J. L. R., Ghosh, D., \& Strong, K. T. 1996, ApJ, 473, 519

Strong, K. 1978, PhD Thesis, University College London, UK

Thomas, R. J., \& Neupert, W. M. 1994, ApJS, 91, 461

Tyrén, F. 1938, Z. für Physik, 111, 314

Witthoeft, M. C., Badnell, N. R., del Zanna, G., Berrington, K. A., \& Pelan, J. C. 2006, A\&A, 446, 361

Young, P. R., Del Zanna, G., Landi, E., et al. 2003, ApJS, 144, 135 